\title{
Exponential Time Differencing Method for Studying Prey-Predator Dynamic during Mating Period
}

\author{
Noufe H. Aljahdaly $\mathbb{D}^{1}$ and H. A. Ashi $\mathbb{1 D}^{2}$ \\ ${ }^{1}$ Department of Mathematics, Faculty of Sciences and Arts-Rabigh Campus, King Abdulaziz University, Jeddah, Saudi Arabia \\ ${ }^{2}$ Department of Mathematics, Faculty of Science, King Abdulaziz University, Jeddah 21589, Saudi Arabia
}

Correspondence should be addressed to Noufe H. Aljahdaly; nhaljahdaly@kau.edu.sa

Received 25 May 2021; Accepted 24 August 2021; Published 21 September 2021

Academic Editor: Chung-Min Liao

Copyright (c) 2021 Noufe H. Aljahdaly and H. A. Ashi. This is an open access article distributed under the Creative Commons Attribution License, which permits unrestricted use, distribution, and reproduction in any medium, provided the original work is properly cited.

\begin{abstract}
This paper addresses a first numerical simulation to the nonlinear dynamic system of equations that describes the prey-predator model at the predator mating period. Some male species accompany the females during the mating period. In this case, both male and female feed on the same prey. The presented work shows the numerical solution for this specific case of the prey-predator mathematical model via an exponential time differencing method. In addition, the paper provides the biological implication of the solution.
\end{abstract}

\section{Introduction}

Many applications and phenomena in physics, biology, the medical field, and other fields can be described as a mathematical model in order to understand or predict the dynamic of these phenomena or applications [1-3]. The prey-predator model is a famous biomathematical model that has been investigated widely to study the dynamic of the ecology system and an intersection between prey and predator [4]. The LotkaVolterra model is known as the predator-prey model for the study population process [5-7]. The model has been studied when considering the influence of diseases [8], prey refuge [9], food chain [10], or competitive populations [11]. The model also was developed to study the intersection between two different populations of predators and one prey $[12,13]$. Recently, the model was modified to study the intersection between two predators from the same species (female and male) and one prey. In some species, the male stays with the female all the time, and they feed together at the mating period. This model is given as follows [14]:

$$
\begin{aligned}
& u_{t}=a_{1} u-a_{2} u^{2}-a_{3} u v-a_{4} u v^{2}, \\
& v_{t}=D_{2}(\Delta v)+a_{3} u v-a_{5} v-a_{6} v^{2}+a_{4} u v^{2},
\end{aligned}
$$

where $u$ and $v$ are the total numbers of prey and predator, respectively. $a_{1}$ is the prey's growth rate. $a_{2}, a_{3}$, and $a_{4}$ are the prey's decay rate due to competition on food supply between the male and the female, between one predator and one prey, and between two predators and one prey, respectively. $a_{5}$ is the mortality rate for the predator. $a_{6}$ is the decay rate of the predator due to competition on the food supply between the male and the female. $D_{2}$ is the predator's diffusion term. In Reference [14], the system (1) was rescaled and became a dimensionless nonlinear system as follows:

$$
\begin{aligned}
& U_{t}=U-U^{2}-U V-U V^{2}, \\
& V_{t}=V_{X X}-k_{2} k_{1} V-k_{1} V^{2}+k_{1} V U+k_{1} U V^{2},
\end{aligned}
$$

where $k_{1}=\sqrt{a_{4} a_{1}} / a_{2}=a_{3} / a_{2}$ and $k_{2}=a_{5} /\left(a_{1} k_{1}\right)$.

The mathematicians put forth more effort in the computational field to improve different techniques to be able to find the consistent solutions for realistic applications, for example, the geometric-qualitative method, semiapproximate method, qualitative method, numerical method, and analytical method. Analytical methods generate different solutions based on many factors such as used ansatz. There 

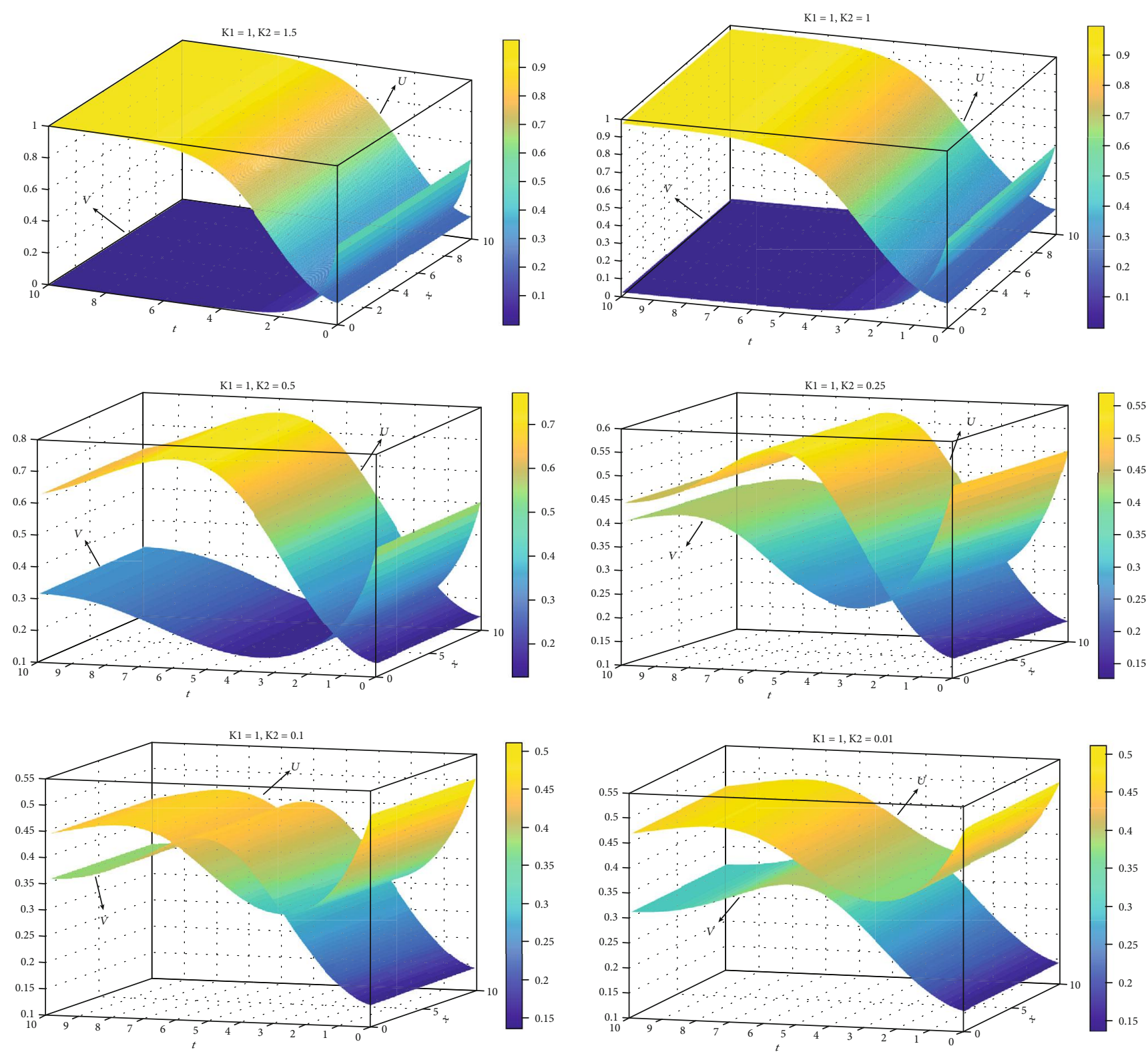

Figure 1: Plot of $U, V$ for $k_{1}=1$ and $0<k_{2}<(1+\sqrt{5}) / 2$.

are many methods to obtain the analytical solutions such as the $\left(G^{\prime} / G^{2}\right)$-expansion approach [15] and the direct algebraic method [16-19]. They are able to obtain the solutions without an initial condition. Each method can introduce new solutions to the system. The solutions of system (2) were found by two analytical methods, the $\left(G^{\prime} / G^{2}\right)$-expansion approach and the direct algebraic method [15]. Since the analytical methods give different solutions, it is difficult to determine which solution predicts the applications. On the other hand, the numerical method for the initial value problem is the method that finds the approximate solution and converges to only one solution based on the initial condition that is obtained from the application, for example, the finite difference method [20-22], Adomian decomposition method (ADM) [23], Laplacian Adomian decomposition method (LADM), multistage differential transform method $[24,25]$, and exponential time differencing method [26]. The numerical solution for system (2) will be introduced in this paper via the exponential time differencing method since the method is fourth-order accurate and is not local convergent comparing to ADM or LADM. The results either determine which analytical solution that predicts the problem or find a new solution.

The paper is organized as follows: the next section presents the numerical results and the scheme of the used method, the third section discusses the numerical solution from the biological perspective, the fourth section is a comparison with the previous studies for the aforementioned model, and the last section is the summary of our analysis and results. 


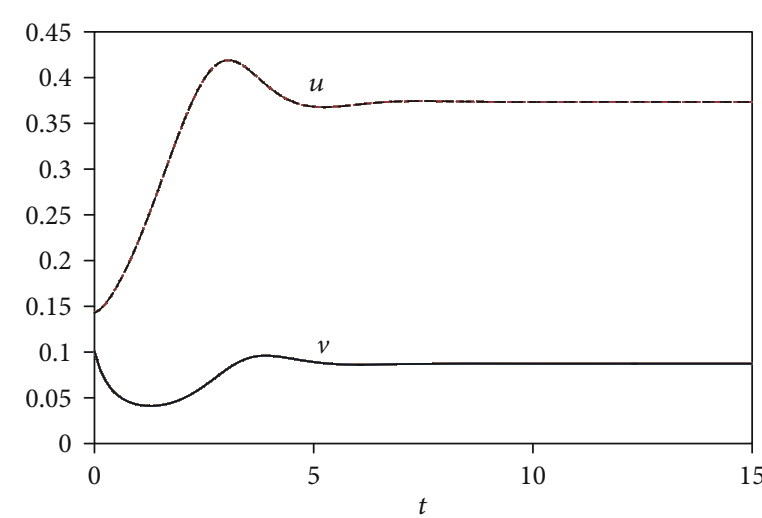

(a) $a_{4}=10$

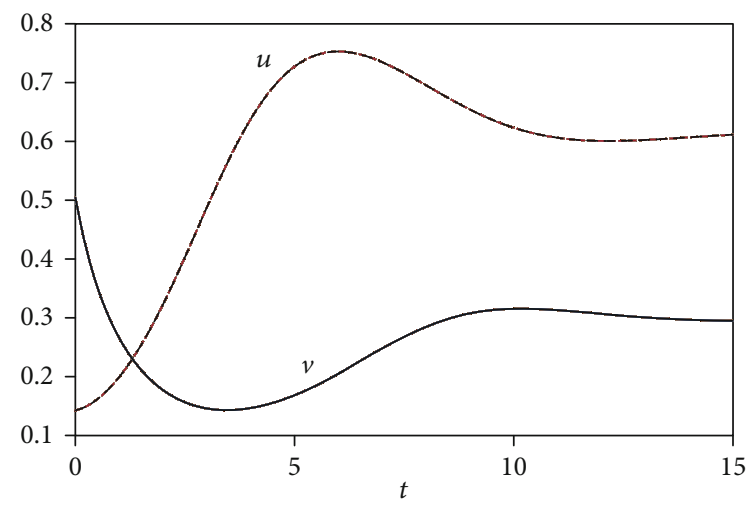

(c) $a_{4}=1$

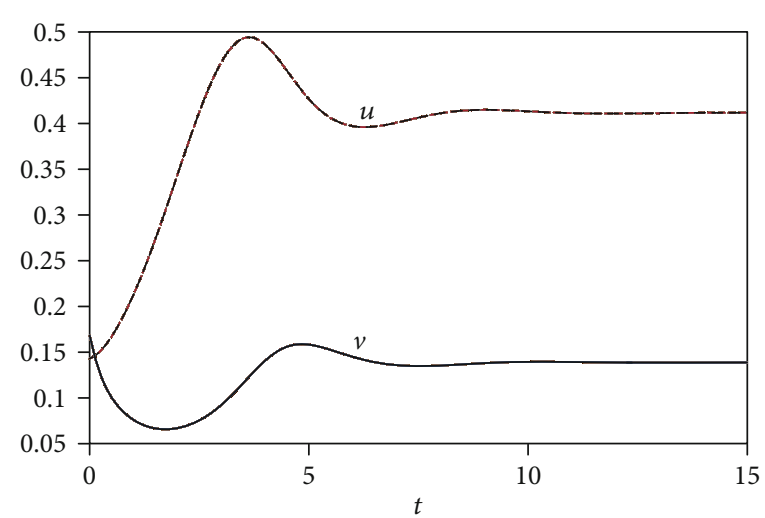

(b) $a_{4}=5$

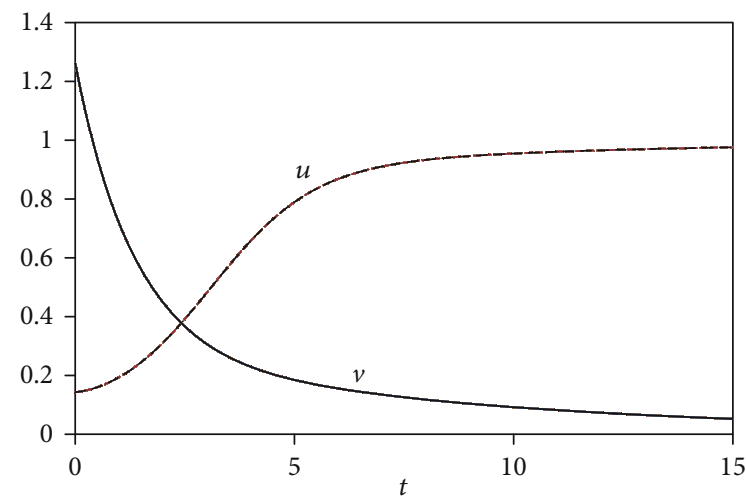

(d) $a_{4}=0.4$

Figure 2: Plot of $u, v$ for $a_{1}=a_{2}=1, a_{5}=0.5$ to capture the effect of the term $a_{4} u v^{2}$ on the model.

\section{Numerical Simulation}

In this section, we give the approximate numerical solution of system (2). First, the system is discretized in space utilizing the Fourier spectral method. Hence, we obtain the following system of ordinary differential equations (ODEs) that depends only on time:

$$
\begin{aligned}
& \frac{d \widehat{u}_{k}(t)}{d t}=\widehat{u}_{k}(t)+\widehat{F}_{k}\left(\widehat{u}_{k}(t), \widehat{v}_{k}(t), t\right), \\
& \frac{d \widehat{v}_{k}(t)}{d t}=c \widehat{v}_{k}(t)+\widehat{G}_{k}\left(\widehat{u}_{k}(t), \widehat{v}_{k}(t), t\right),
\end{aligned}
$$

where the parameter $c=k^{2}-k_{2} k_{1}$ is associated to the linear part, $k$ represents the wave numbers, both terms

$$
\begin{aligned}
& \left.\widehat{F}_{k}\left(\widehat{u}_{k}(t), \widehat{v}_{k}(t), t\right)\right)=f f t\left(-U^{2}(t)-U(t) V(t)-U(t) V^{2}(t)\right), \\
& \left.\widehat{G}_{k}\left(\widehat{u}_{k}(t), \widehat{v}_{k}(t), t\right)\right)=k_{1} f f t\left(-V^{2}(t)+U(t) V(t)+U(t) V^{2}(t)\right),
\end{aligned}
$$

represent the nonlinear forcing term, and $\mathrm{fft}$ is the MATLAB command that represents the fast Fourier transform (FFT).

Second, we integrate the obtained uncoupled system of ODEs (3) in time with the aid of the fourth-order exponential time differencing method ETD4RK [27-29]. Applying the ETD4RK method to (3b) takes the formula

$$
\begin{aligned}
& a_{k, n}=\widehat{v}_{k, n} e^{c \Delta t / 2}+\left(e^{c \Delta t / 2}-1\right) \frac{\widehat{G}_{k, n}}{c}, \\
& b_{k, n}=\widehat{v}_{k, n} e^{c \Delta t / 2}+\frac{\left(e^{c \Delta t / 2}-1\right) \widehat{G}\left(a_{k, n}, t_{n}+\Delta t / 2\right)}{c}, \\
& C_{k, n}=a_{k, n} e^{c \Delta t / 2}+\frac{\left(e^{c \Delta t / 2}-1\right)\left(2 \widehat{G}\left(b_{k, n}, t_{n}+\Delta t / 2\right)-\widehat{G}_{k, n}\right)}{c},
\end{aligned}
$$

$$
\begin{aligned}
\widehat{v}_{k, n+1}= & \widehat{v}_{k, n} e^{c \Delta t}+\left(\left(c^{2} \Delta t^{2}-3 c \Delta t+4\right) e^{c \Delta t}-c \Delta t-4\right) \widehat{G}_{k, n} \\
& +2\left((c \Delta t-2) e^{c \Delta t}+c \Delta t+2\right)\left(\widehat{G}\left(a_{k, n}, t_{n}+\frac{\Delta t}{2}\right)\right. \\
& \left.+\widehat{G}\left(b_{k, n}, t_{n}+\frac{\Delta t}{2}\right)\right) \\
& +\frac{\left((-c \Delta t+4) e^{c \Delta t}-c^{2} \Delta t^{2}-3 c \Delta t-4\right) \widehat{G}\left(C_{k, n}, t_{n}+\Delta t\right)}{c^{3} \Delta t^{2}},
\end{aligned}
$$

where $\Delta t$ represents the time step (a similar formula is obtained when applying the ETD4RK method to (3a) with $c=1)$. 


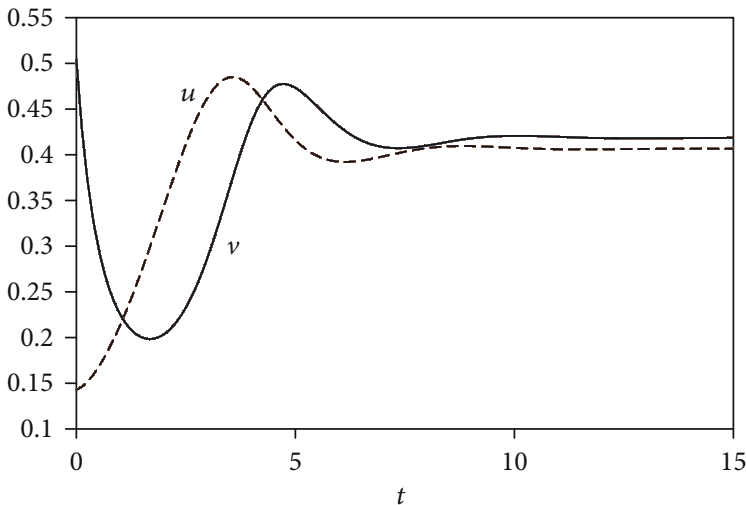

(a) $a_{3}=5$

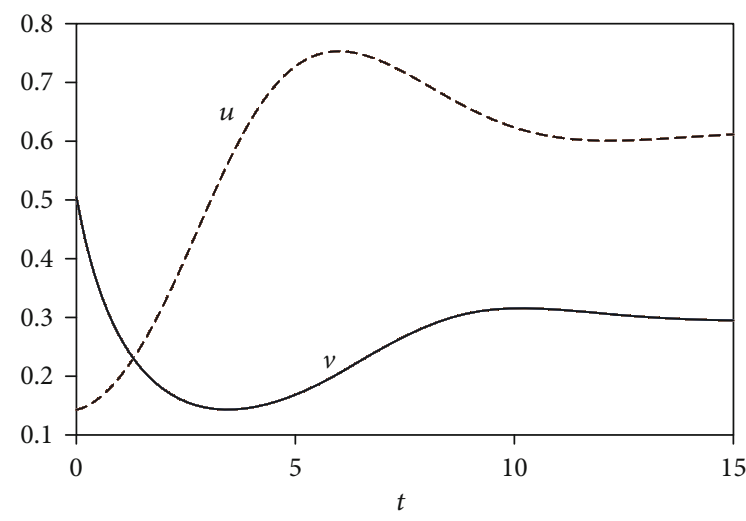

(c) $a_{3}=1$

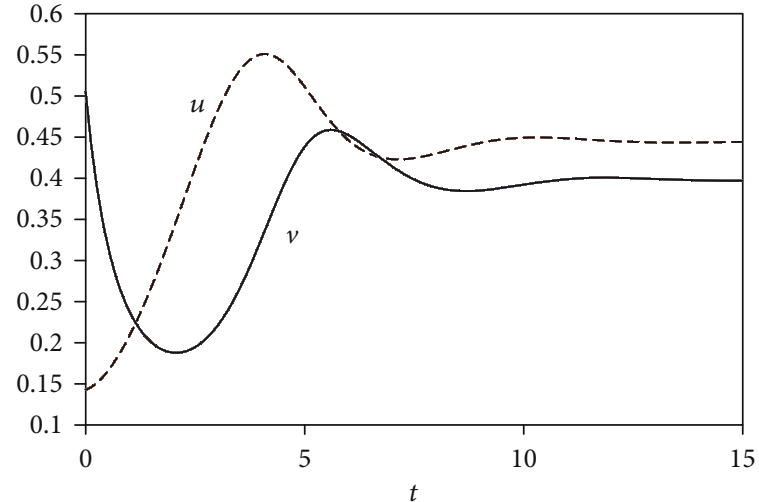

(b) $a_{3}=3$

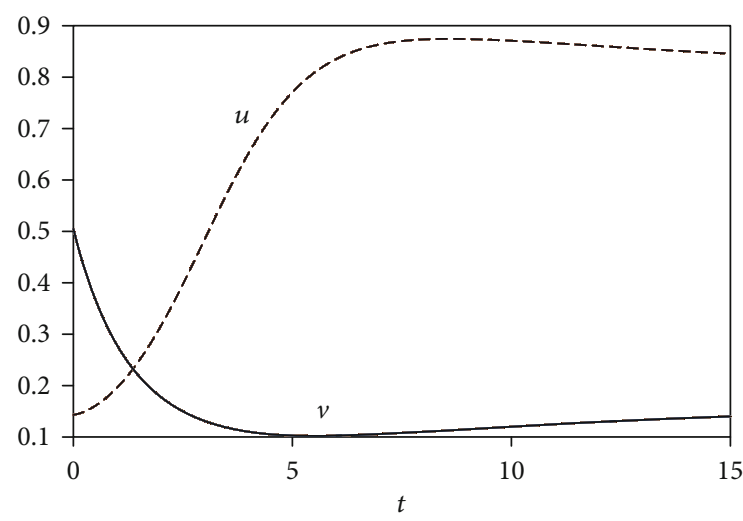

(d) $a_{3}=0.4$

FIgURE 3: Plot of $u, v$ for $a_{1}=a_{2}=1, a_{5}=0.5$ to capture the effect of the term $a_{3} u v$ on the model.

The time integration starts with initial conditions (taken from [30]) given by

$$
\begin{aligned}
& \widehat{u}_{0}=\frac{6}{35}-2 \times 10^{-7}(x-225)(x-675), \\
& \widehat{v}_{0}=\frac{116}{245}-3 \times 10^{-5}(x-450)+180 \times 10^{-4} .
\end{aligned}
$$

The results of our experiment are shown in figures.

The solution is stable at $0 \leq k_{2} \leq(1+\sqrt{5}) / 2$ based on the stability analysis in Reference [14]. A numerical solution is produced in Figure 1 for different value of $k_{2}$. We realized that solutions are changed with $k_{2}$. When $k_{2}=1.5, U$ increases while $V$ decreases until they reach the steady state. For $k_{2}=0.1$, the solution is periodic. The parameter $k_{2}=$ $\left(a_{2} a_{5}\right) /\left(a_{1} \sqrt{a_{4} a_{1}}\right)=\left(a_{5} a_{2}\right) /\left(a_{1} a_{3}\right)$ has direct relation with $a_{5}$ and $a_{2}$ and negative relation with $a_{1}, a_{3}$, and $a_{4}$.

\section{Biological Relevance of the Solution}

A new term in the studied model is $a_{4} v^{2} u$. Thus, the numerical solution is used to study the effect of this term on a traditional prey-predator model. The solution of the traditional model is an undamped oscillation, and Lotka explained this behavior because of the law of mass action [5], while the presented model (2) has an oscillation solution with small $k_{2}$ at a bounded space and the solution converges to the steady state out of this space, because $a_{4} v^{2} u$ is related to the interaction between prey and two predators at the mating period which disappears after this period and also happens in a specific location where the predators give birth.

The large rate of $a_{4}$ indicates that the predators feed on a large amount of prey in this period, which lead to a large size of the predator population than the prey population. This is not an optimal dynamic of population to live normally (see Figure 2 when $a_{4}=10$ ). Therefore, the predator population will decrease because of absence of a food source, while the prey population will grow again. The new term $a_{4} v^{2} u$ has more effect than $a_{3} u v$ to decay the prey population as we see by comparing Figures 3 and 2 .

\section{Connection with the Previous Studies}

The analytical solutions of Equations (2) was found by Aljahdaly and Alqudah [31] using $\left(G^{\prime} / G\right)$ and the generalized auxiliary equation [15]. The solutions are $U(x, t)$ and $V(x, t)$ which denote the population of the prey and predator, respectively, in a single patch. The solutions predict the two following behaviors

4.1. The $\left(G^{\prime} / G\right)$-Expansion Method (Solution (1)). As a result of two predators' sexual interaction and the high prey consumption during the predator's mating period, $V$ increases 
and $U$ decreases. Ultimately, $V$ will become dominant in the patch.

4.2. Generalized Auxiliary Equation Method (Solution (2)). The predators graze in the mating period where the prey density $(U)$ is large. Then, $U$ decrease and $V$ increase. At a certain time, $U$ will be very low, and the predators will either die or move to different patches. Then, the prey population will recover and grow again in the same location.

4.3. Exponential Time Differencing Method. For a large value of $k_{2}$, we obtain the numerical solution that converges to solution (1), while for small $k_{2}$, the numerical solution approximates solution (2).

The numerical solution gives the important reasons behind the two different solutions by the analytical methods.

\section{Conclusion}

The prey-predator system with the extra term $a_{4} v^{2} u$ (1) is a new nonlinear dynamic system of equations in the literature. It has been solved analytically with two different methods which give two different solutions. The importance of this paper is that the numerical solution gives connection between the obtained analytical solutions from the biological perspective. The numerical solution predicts the dynamic of the studied model as well as proves the effect of the new extra term on the model.

\section{Data Availability}

The data supporting this research are from previously reported studies, which have been cited.

\section{Conflicts of Interest}

The authors declare that there are no conflicts of interest regarding the publication of this paper.

\section{References}

[1] R. M. Al-Maalwi, S. Al-sheikh, H. A. Ashi, and S. Asiri, "Mathematical modeling and parameter estimation of unemployment with the impact of training programs," Mathematics and Computers in Simulation, vol. 182, pp. 705-720, 2021.

[2] R. M. Al-Maalwi, H. Ashi, and S. Al-sheikh, "Unemployment model," Applied Mathematical Sciences, vol. 12, no. 21, pp. 989-1006, 2018.

[3] N. H. Aljahdaly and R. Alharbey, "Fractional numerical simulation of mathematical model of HIV-1 infection with stem cell therapy," AIMS Mathematics, vol. 6, no. 7, pp. 67156725, 2021.

[4] A. A. Berryman, "The origins and evolution of predator-prey theory," Ecology, vol. 73, no. 5, pp. 1530-1535, 1992.

[5] A. J. Lotka, "Elements of physical biology," Science Progress in the twentieth century (1919-1933), vol. 21, pp. 341-343, 1926.

[6] Y. Takeuchi, Global Dynamical Properties of Lotka-Volterra Systems, World Scientific, 1996.

[7] V. Volterra, "Variations and fluctuations of the number of individuals in animal species living together," Animal ecology, pp. 409-448, 1926.
[8] E. Venturino, "The influence of diseases on Lotka-Volterra systems," The Rocky Mountain Journal of Mathematics, pp. 381-402, 1994.

[9] Q. Wang, Z. Liu, X. Zhang, and R. A. Cheke, "Incorporating prey refuge into a predator-prey system with imprecise parameter estimates," Computational and Applied Mathematics, vol. 36, no. 2, pp. 1067-1084, 2017.

[10] D. Sahoo and G. Samanta, "Comparison between two tritrophic food chain models with multiple delays and antipredation effect," International Journal of Biomathematics, vol. 14, no. 3, article 2150010, 2021.

[11] J. Bao, X. Mao, G. Yin, and C. Yuan, "Competitive LotkaVolterra population dynamics with jumps," Nonlinear Analysis: Theory, Methods \& Applications, vol. 74, no. 17, pp. 6601-6616, 2011.

[12] S. Sarwardi, P. K. Mandal, and S. Ray, "Dynamical behaviour of a two-predator model with prey refuge," Journal of Biological Physics, vol. 39, no. 4, pp. 701-722, 2013.

[13] I. Sayekti, M. Malik, and D. Aldila, "One-prey two-predator model with prey harvesting in a food chain interaction," in AIP Conference Proceedings, Depok, Jawa Barat, Indonesia, 2017.

[14] M. Al Qudah, "Existence, uniqueness solution of a modified predator-prey model," Nonlinear Analysis and Differential Equations, vol. 4, pp. 669-677, 2016.

[15] N. H. Aljahdaly, "Some applications of the modified (G'/G2)expansion method in mathematical physics," Results in Physics, vol. 13, article 102272, 2019.

[16] W. A. Aibarakati, A. R. Seadaw, and N. H. Aljahdaly, "Application of mathematical methods for the non-linear seventh order Sawada-Kotera-Ito dynamical wave equation," Thermal Science, vol. 23, Suppl. 6, pp. 2081-2093, 2019.

[17] N. H. Aljahdaly, A. R. Seadawy, and W. A. Albarakati, "Applications of dispersive analytical wave solutions of nonlinear seventh order lax and Kaup-Kupershmidt dynamical wave equations," Results in Physics, vol. 14, article 102372, 2019.

[18] N. H. Aljahdaly, A. R. Seadawy, and W. A. Albarakati, "Analytical wave solution for the generalized nonlinear seventh-order KDV dynamical equations arising in shallow water waves," Modern Physics Letters B, vol. 34, no. 26, article 2050279, 2020.

[19] A. Seadawy, A. Ali, and N. Aljahdaly, "The nonlinear integrodifferential Ito dynamical equation via three modified mathematical methods and its analytical solutions," Open Physics, vol. 18, no. 1, pp. 24-32, 2020.

[20] M. Ismail and H. Ashi, "A numerical solution for HirotaSatsuma coupled KDV equation," Abstract and Applied Analysis, 615 pages, 2014.

[21] M. Ismail and H. Ashi, "A compact finite difference schemes for solving the coupled nonlinear Schrodinger-Boussinesq equations," Applied Mathematics, vol. 7, no. 7, pp. 605-615, 2016.

[22] M. Ismail, H. Ashi, and F. Al-Rakhemy, "ADI method for solving the two-dimensional coupled nonlinear Schrodinger equation," in AIP Conference Proceedings, Rhodes, Greece, 2015.

[23] N. H. Aljahdaly and S. El-Tantawy, "Simulation study on nonlinear structures in nonlinear dispersive media," Chaos: An Interdisciplinary Journal of Nonlinear Science, vol. 30, no. 5, article 053117, 2020. 
[24] N. H. Aljahdaly, "New application through multistage differential transform method," in AIP conference proceedings, Rhodes, Greece, 2020.

[25] N. H. Aljahdaly and S. El-Tantawy, "On the multistage differential transformation method for analyzing damping duffing oscillator and its applications to plasma physics," Mathematics, vol. 9, no. 4, p. 432, 2021.

[26] H. A. Ashi and N. H. Aljahdaly, "Breather and solitons waves in optical fibers via exponential time differencing method," Communications in Nonlinear Science and Numerical Simulation, vol. 85, article 105237, 2020.

[27] H. Ashi, L. Cummings, and P. Matthews, "Comparison of methods for evaluating functions of a matrix exponential," Applied Numerical Mathematics, vol. 59, no. 3-4, pp. 468486, 2009.

[28] H. Ashi, "Solving stiff reaction-diffusion equations using exponential time differences methods," American Journal of Computational Mathematics, vol. 8, no. 1, pp. 55-67, 2018.

[29] S. M. Cox and P. C. Matthews, "Exponential time differencing for stiff systems," Journal of Computational Physics, vol. 176, no. 2, pp. 430-455, 2002.

[30] M. R. Garvie, "Finite-difference schemes for reaction-diffusion equations modeling predator-prey interactions in MATLAB," Bulletin of Mathematical Biology, vol. 69, no. 3, pp. 931-956, 2007.

[31] N. H. Aljahdaly and M. A. Alqudah, "Analytical solutions of a modified predator-prey model through a new ecological interaction," Computational and mathematical methods in medicine, vol. 2019, 7 pages, 2019. 